\title{
Why Europe Needs Public Funding for Platform Development
}

\author{
Paul Nemitz and Arndt Kwiatkowksi*
}

\section{Introduction}

Over the last decade, Google and Facebook from the US and Tencent and Alibaba from China, have established leading global platforms in the general 'Business to Consumer' markets. They were able to do so in huge home markets without language barriers. They also benefitted from a first mover advantage, low regulatory hurdles, the scalability of their business model and the almost unlimited availability of capital. In their home markets these platforms seem to be nearly unchallenged by competition. In Europe, Google has a market share for search of more than 90\%. After its acquisition of WhatsApp and Instagram, Facebook seems to be equally unchallenged in its strong market position. However, in their home markets these platforms have also not yet been subject to effective application of competition law.

The 'winner takes all' mechanism these platforms benefit from is referred to as the network effect. The value of the platform to users is really created by all the other participants (eg users, app-developers, vendors) on that network: A user's decision of not using the leading platform creates disadvantages, eg lack of choice.

The network effect enables the leading platforms to achieve massive scale and eliminate competition. Many of the platform markets tend naturally toward monopolies. The network effect makes it basically impossible to establish a competitor once a dominant platform exists because switching costs for all users on all sides of the platform are too high and the smaller number of followers can't populate the competing network sufficiently to make them attractive as a challenger.

DOI: 10.21552/delphi/2019/2/9

* Paul Nemitz is Principle Advisor in the EU Commission and a Member of the German Federal Government's Data Ethics Commission. He is expressing his personal opinion. Arndt Kwiatkowksi is the Co-founder and CEO of Bettermarks.com, the digital education service for mathematics in school systems.
That is why time is of the essence for the creation of a lively ecosystem, when it comes to setting up platforms for new markets. And there is still space for other, more specialised 'Business to Business' (B2B) platforms to be set up in Europe which could potentially be scaled beyond Europe.

In the following, we will look at the potential for B2B platform development in Europe in three sectors. We look at sectors which are not yet covered by dominant platforms in Europe and in which, at the same time, governments have a strong position in terms of their ability to shape conditions for market development. Specifically we examine the markets for services in public administration, education and health. Here, international innovation leaders from Europe can emerge, if the EU and Member States lower the hurdles for innovative application development and provide targeted financial support for this purpose, in line with the strong public interest in shaping these markets.

The condition for establishing European platforms successfully in these markets are good:

- The benefits of digitisation and the platform model in these three markets are enormous and have hardly been realised so far.

- Dominant platforms do not exist in these three areas, neither in Europe nor internationally.

- Access to users and data as well as the use of platforms in these fields can be efficiently promoted through public authorities, given that they are the sole buyers for administration services and in most EU Member States have a strong hold on education and health services, both as regulators and as buyers.

- Private investment in platforms in these three markets in Europe has been scarce, due to the strong position of governments in these markets and also due to the fragmentation of requirements and languages, which is of greatest relevance in the education and administration markets.

- Investment funds needed for the support of innovative initiatives to create platforms in Europe are probably in the 2-digit millions per sector. This can 
and should be mobilised by the EU and its Member States, as risk capital is lacking in these segments.

- The development of platforms for high-quality services in the fields of health, education and public administration is both in the public interest and a basis for European and possibly global scaling, and thus profitability, which in turn enables further improvement of the service at home and also secures growth and employment in Europe.

With the continuing development of 'Industry 4.0' (Internet of Things) more platforms will emerge in other sectors (eg agriculture, segments of mechanical engineering, transport). However, these will be sector-specific and in comparison to the above mention markets, the potential for government influence is lower. These are also markets for which more capital is available.

Platform companies are not only relevant from the point of view of 'citizen benefit' but also in terms of the potential economic reward. Experience has shown that platform-based services are often developed in the US and then also offered in Europe. However, it would be desirable to promote the development of platforms in Europe, as this is the only way to implement our legal and value concepts (eg data protection, transparency) from the outset and 'by design' and to profit economically from international market leaders for services relating to health, administration and education based in Europe. This would also allow the products to be tailored to the specific domestic requirements of the EU and its Member States.

There are a number of European projects and programmes which have already prepared the ground for successful initiatives on platforms in Europe, both in the form of sectoral initiatives (for example the 'Digital Learning \& ICT Education' work stream of the European Commission) as well as horizontal work relating to issues like user control over personal data (Decodeproject.eu).

\section{Critical Factors}

The critical factors for platforms in these markets include the lowering of innovation hurdles, a rapid spread of data use and sufficient financing in the early expansion phase. Accordingly, cooperation with institutional users and the availability of data and open source code are essential for lowering innovation hurdles.

\section{Health}

The healthcare sector is a good illustration of this. For instance, the availability of anonymised data (eg relevant partial information from images from CTG and X-Ray scans) is vital in order to train algorithms. Without these, the hurdle to innovative application development is enormous. A further hurdle is the availability of case numbers and appointment calendars. With that in mind partnerships between developers and actors in the healthcare sector should be encouraged through targeted support and guidance as to the proper implementation of the GDPR.

The availability of APIs to conduct diagnostic functions (as developed by the App 'Ada health') could be an excellent basis to develop further applications for the prioritisation of physician appointments, for example. If the results of the diagnostic tool were linked and compared with actual findings, the prognosis quality of the app based diagnostics tool could be continuously improved. It could also be used to develop applications for the quality assurance of medical work.

More advanced applications for the evaluation of images and measurement results such as smart radiology could be developed if the data from hospitals was accessible, possibly as part of development partnerships, through which full compliance with GDPR can be ensured.

\section{Public Administration}

Pertinent examples from public administration include the availability of mobility data from public transport, rail, air transport and private car use. With free access to this data in anonymised form, suitable mobility offers and traffic control systems could be developed that allow citizens and municipalities to incorporate the respective individual or democratically determined preferences in the region, which is not possible with global applications such as Google Maps or Waze.

It would also be desirable that more administrative services are provided to citizens online. The digi- 
tisation of more than 500 administrative processes between state and citizens is already an ongoing project of the German government with the federal states and municipalities sharing the tasks in an organised division of labour. This would be facilitated by a digital identity as has been developed in Estonia. Building on this, 'Identity Guaranteed Administrative Platforms' or 'Trust Platforms' can be created, which are also needed internationally in order to offer administrative services (car re-registration, extension of identity card, unemployment registration, etc). Health data can be stored for doctors to retrieve (living will, organ donation requests). The identity and administrative platforms can also serve as a secure platform for the processing of transactions of third parties, where the identification of the identity is important (account opening, car purchase, transmission of documents, etc). The legal basis for this already exist in EU law.

\section{Education}

In the field of education, the free availability of tools for creating adaptive learning materials is a prerequisite for the development of innovative learning media. Since the development of an 'adaptive learning platform' is technically complex and costs tens of millions of Euros, the hurdle would be too high for most content providers if they had to develop their own proprietary platform. On the other hand, access to a common platform with easy-to-use authoring tools that is provided by the state, enables educational providers to transfer their didactic knowledge into effective digital learning media without high investment cost. In addition, the competition for innovative applications would be further accelerated if the usage data were made available to the public in an anonymous format, or at least to the participants of the platform, so that experience from different learning processes and applications could be used repeatedly to improve the quality of service and ease of use.

In addition to financing the platform (provision as open source code) and access to usage datasets, policymakers could create further framework conditions to promote the ecosystem around the platform. For example, suitable platform offerings could be available as part of larger curricula, eg a financial accounting course could be recognised as equivalent to a face-to-face MBA course, thus increasing the permeability, flexibility and capacity of the education system.

A digital platform for lifelong learning would also be a logical next step based on the development of public databases of learning materials for teachers and schools.

The public school system in Europe, which is democratically controlled, needs its own publicly controlled digital learning platform for digital educational content. Such a learning platform can also become an international standard. Conversely, neither the quality nor the democratic control of learning content in the EU and its Member States can be maintained in the long term if we leave the development and provision of such a learning platform to the global market. By now we should know that only those who master the infrastructure of digital services can also control the content and its quality.

One challenge for the education system is the 'mismatch' of unplaced jobseekers and unfilled vacancies for skilled workers. There is an 'education gap' both from the individual perspective of the job seeker and from the perspective of the economy, which complains of a shortage of skilled workers. In the technical professions, the mismatch problem becomes an obstacle to investment, as SMEs in particular do not invest in new technologies if they are not sure that they can quickly hire suitably qualified employees who can use the new technologies, or that they can offer or purchase appropriate training themselves.

In school education, the biggest problem is the lack of teachers and the development of students' performance in the MINT subjects. This is where there is most experience with digital learning systems that can remedy the situation.

Europe should not wait for the next generation of platform companies for educational content from the USA or China (comparable to the Android or Apple App Store) to establish themselves as the market standard. It rather makes sense to enable such a platform for the European education systems through state or EU startup financing and anchoring in or close to the state educational institutions. The advantages are:

- Efficient and up-to-date learning media are developed in a wide variety in a timely manner

- Data protection aspects, which play an important role with regard to competence profiles of learners, can be integrated. 
- Direct access to educational content for all citizens and education systems

- Internationalisation opportunities for platform and educational content from Europe

- Development parallel to curricula and teaching and study methods common here

- Integration in teacher training and further education

\section{Conclusion}

In all markets that are changed by digitalisation, digital projects are successful if they are continuously improved and their impact is measured from the point of use. In the three markets mentioned, there is great potential for policy to influence the creation of applications in these areas.

The measurable benefit and rapid dissemination in the home market is then in turn the prerequisite for successful internationalisation, and this in turn forms the basis for further improvement of the service. The positive correlation between scaling and quality development is visible across all platforms, starting with Google. Europe and its Member States can benefit directly from these effects if the state and industry work together to ensure that platforms are created in Europe for the aforementioned purposes. This requires a targeted, coordinated lead market and promotion policy on the part of the EU and the Member State's governments. But there is also space for Member State initiatives, in particular in the fragmented education sector. 